\title{
Reliability Analysis Based on Coupled Relationship
}

\author{
W.L. Pan \\ School of Mathematics \& Computer Science \\ Yunnan Minzu University \\ Yunnan, China \\ Key Laboratory of IOT Application Technology of \\ Universities in Yunnan Province \\ Yunnan Minzu University \\ Yunnan, China
}

\author{
H. Wang \\ College of Mechanical and Electrical Engineering \\ Harbin Engineering University \\ Heilongjiang, China
}

\begin{abstract}
In the usual reliability analysis, researchers often acquire the reliability information about a product by reliability tests and usually regard the product as a whole object to study. Also, the relationships between the attributes of the product are usually ignored. Base on the non-IIDness learning, the attributes of objects are more or less interacted and coupled. Thus, this paper analyzes the coupled relationships between each pair of product attributes and the coupled relationships between each product attribute and reliability lifetime of the product. Specifically, the attributes we analyzed are continuous. The level of interplay between each pair of attributes and between each attribute and product lifetime can be observed by coupled correlation matrices.
\end{abstract}

Keywords-reliability; non-IIDness learning; coupled relationship

\section{INTRODUCTION}

Reliability is defined as the probability that a device will perform the intended function under stated condition for a specified period of time. Reliability technology is a technology for improving the reliability of products, it studies on the failure reasons of a system or product and measures should be taken to eliminate and prevent the failure. The aim of this technology is to ensure the reliability and availability of a product, prolong the lifetime of using, decrease the maintenance costs and improve the utility of a product. Many factors may result in the failure of a product.Thus, it's hard to predict the reliability of a product. In real production, we often use the probability and statistics theory to evaluate the reliability of the product.

Reliability data is the basis of reliability engineering and the analysis object of reliability technology. In the process of planing, designing, manufacturing, using and maintaining a product, it produces a large amount of data which is vital for analyzing the reliability. There are five methods to get the reliability data: direct collection, questionnaire, regular feedback and reliability tests. Especially reliability tests is a common and effective method.

According to the test purposes, reliability tests can be divided into two types, one is engineering tests, another one is statistical tests. The aim of engineering tests is to reveal the drawbacks about design, crafts and the material of a product, then give feedback to the design and production department to improve the reliability of a product. The aim of statistical test is to verify whether the reliability of a product reaches the standards, rather than to know the drawbacks of a product.

Generally speaking, when we are making a traditional analysis of reliability, we build a statistical model based on experimental data and predict the product lifetime under the desirable reliability by verifying the failure probability and the distribution of lifetime. However, in the practical situation, according to non-IIDness theory [1], the attributes of objects are more or less interacted and coupled. As Figure 1 shows, the different attributes impact each other. So do the product lifetime and the product attributes.

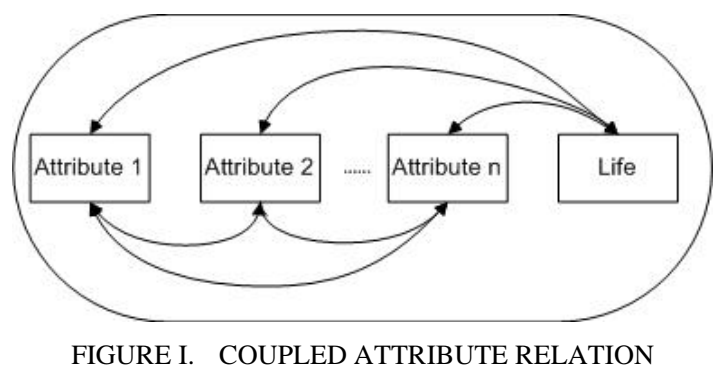

Therefore, during making a traditional analysis of reliability, if we regard the object as a whole object regardless of the coupled relation between the object attributes, the following disadvantages will be appeared: (1) The level of interplay between each attribute and reliability lifetime can't be observed clearly. (2) The level of interplay between each pair of attributes can't be observed either.

According to the statistics and data mining theory, after getting amounts of reliability data, we can acquire more information about a product lifetime by data mining. So, based on data mining theory and non-IIDness learning theory, we propose a method to analyze the relationship between each pair of attributes and the relationship between each attribute and reliability lifetime, where the attributes are continuous.

\section{RELATED WORK}

Reliability lifetime prediction and analysis has always been the focus of reliability engineering. There are many different kind of methods to predict reliability lifetime. The life prediction method based on stress is the earliest method and the most common method [2]. Meanwhile, with the development of artificial intelligence technology, a number of new and efficient method for life prediction were invented. 
Such as the prediction of life based on neural networks, the prediction of life based on expert systems, the prediction of life based on fuzzy systems and the prediction of life based on evolutionary algorithms [3]. The factors which effect the reliability life are complex. After obtaining the reliability life data we can analyze the data further through data mining and expect to acquire the relationship between attributes and life. $\mathrm{L}$. Cao proposed the non-IIDness learning which thinks attribute values and attributes are usually coupled [1]. Wang et al. put forward the coup nominal similarity in unsupervised learning [4], and analyzed coupled attribute on numerical data [5]. Cheng et al. analyzed the coupled term-term relation for document clustering [6]. Based on these studies about coupled attributes, this paper analyzes the coupled relationships about product attributes and product lifetime, which have been ignored before.

\section{COUPLED RELATIONSHIP ANALYSIS ON ATTRIBUTES AND LIFETIME}

On the basis of reliability tests, we can get a large amount of lifetime data. There are many factors which can affect the lifetime of a product. For instance, the reasons which effect the fatigue lifetime of a gear are complex. The load and the roughness concentration both play important roles in the reliability lifetime of the gear. According to the relationship between two attributes can be calculated by Pearson's correlation coefficient, if the attributes which effect the reliability lifetime are continuous, we can calculate the Pearson's correlation coefficient and acquire how much the attributes effect the reliability lifetime.

With the aim of getting the information about the relationship among attributes and lifetime, we focus on the reliability testing data which attributes are continuous. First, we use the reliability testing data to organize an information table $S=<U, A, A_{L}, f>$ [4], where $U=\left\{u_{1}, u_{2} \cdots u_{m}\right\}$ is composed of a set of data objects of the test; $A=\left\{A_{1}, A_{2} \cdots A_{n}\right\}$ is a set of attributes of the data.objects.; $A_{L}$ is the reliability lifetime; $f_{i}\left(u_{k}\right)=v(1 \leq i \leq n$ or $i=L$, $1 \leq k \leq m)$ is an information function which assigns a particular value of each attribute to every testing object, in which $i$ is the number of the $i$-th attribute or reliability lifetime and $u_{k}$ is the k-th object. For instance, Table 1 is an reliability lifetime information table which consists of six objects and four kind of attributes (especially, in order to represent conveniently, the reliability lifetime also is regarded as a special attribute), the attribute value of object $u_{1}$ on attribute $A_{1}$ is $f_{1}\left(u_{1}\right)=a_{1}$ and $f_{4}\left(u_{1}\right)=l_{1}$ signifies the lifetime value of object $u_{1}$ is $l_{1}$.
TABLE I. THE INFORMATION TABLE OF RELIABILITY LIFETIME

\begin{tabular}{|c|c|c|c|c|}
\hline & $A_{1}$ & $A_{2}$ & $A_{3}$ & $A_{L}$ \\
\hline$u_{1}$ & $a_{1}$ & $b_{1}$ & $c_{1}$ & $l_{1}$ \\
\hline$u_{2}$ & $a_{2}$ & $b_{2}$ & $c_{2}$ & $l_{2}$ \\
\hline$u_{3}$ & $a_{3}$ & $b_{3}$ & $c_{3}$ & $l_{3}$ \\
\hline$u_{4}$ & $a_{4}$ & $b_{4}$ & $c_{4}$ & $l_{4}$ \\
\hline$u_{5}$ & $a_{5}$ & $b_{5}$ & $c_{5}$ & $l_{5}$ \\
\hline$u_{6}$ & $a_{6}$ & $b_{6}$ & $c_{6}$ & $l_{6}$ \\
\hline
\end{tabular}

However the Pearson's correlation coefficient only describes the linear relationship between two variables. Indeed, the relationship between different attributes is complex. Then we expand the numerical space spanned by $\mathrm{n}$ continuous attributes with more dimensions, the coupling relationships between continuous attributes can be exposed by new correlation between every two updated attributes [5].

The way to expand the information table is to add new attributes to the original table. Each original attribute will be expanded to new attributes: $\left(A_{j}\right)^{2},\left(A_{j}\right)^{3} \cdots\left(A_{j}\right)^{D}$. The attribute value of $\left(A_{j}\right)^{p}(1 \leq p \leq D)$ is the p-th power of the corresponding value of attribute $A_{j}$. After the expanding, we can get a new updated reliability lifetime information table. For instance, Table 2 is the extended reliability lifetime information table, with $D=2$.

TABLE II. THE EXTENDED INFORMATION TABLE OF RELIABILITY LIFETIME.

\begin{tabular}{|l|ll|ll|ll|ll|}
\hline & $\left(A_{1}\right)^{1}$ & $\left(A_{1}\right)^{2}$ & $\left(A_{2}\right.$ & $\left(A_{2}\right)^{2}$ & $(A$ & $\left(A_{3}\right)^{2}$ & $\left(A_{L}\right.$ & $\left(A_{L}\right)$ \\
\hline$u_{1}$ & $a_{1}$ & $\left(a_{1}\right)^{2}$ & $b_{1}$ & $\left(b_{1}\right)^{2}$ & $c_{1}$ & $\left(c_{1}\right)^{2}$ & $l_{1}$ & $\left(l_{1}\right)^{2}$ \\
\hline$u_{2}$ & $a_{2}$ & $\left(a_{2}\right)^{2}$ & $b_{2}$ & $\left(b_{2}\right)^{2}$ & $c_{2}$ & $\left(c_{2}\right)^{2}$ & $l_{2}$ & $\left(l_{2}\right)^{2}$ \\
\hline$u_{3}$ & $a_{3}$ & $\left(a_{3}\right)^{2}$ & $b_{3}$ & $\left(b_{3}\right)^{2}$ & $c_{3}$ & $\left(c_{3}\right)^{2}$ & $l_{3}$ & $\left(l_{3}\right)^{2}$ \\
\hline$u_{4}$ & $a_{4}$ & $\left(a_{4}\right)^{2}$ & $b_{4}$ & $\left(b_{4}\right)^{2}$ & $c_{4}$ & $\left(c_{4}\right)^{2}$ & $l_{4}$ & $\left(l_{4}\right)^{2}$ \\
\hline$u_{5}$ & $a_{5}$ & $\left(a_{5}\right)^{2}$ & $b_{5}$ & $\left(b_{5}\right)^{2}$ & $c_{5}$ & $\left(c_{5}\right)^{2}$ & $l_{5}$ & $\left(l_{5}\right)^{2}$ \\
\hline$u_{6}$ & $a_{6}$ & $\left(a_{6}\right)^{2}$ & $b_{6}$ & $\left(b_{6}\right)^{2}$ & $c_{6}$ & $\left(c_{6}\right)^{2}$ & $l_{6}$ & $\left(l_{6}\right)^{2}$ \\
\hline
\end{tabular}

According to the extended information table and Pearson's correlation coefficient, the correlation between different attributes is calculated. First, the Pearson's correlation coefficient between $A_{i}$ and $A_{j}$ is formalized as follows:

$$
\operatorname{Cor}\left(A_{i}, A_{j}\right)=\frac{S_{A_{i} A_{j}}}{S_{A_{i}} \times S_{A_{j}}},
$$

Where

$$
\begin{aligned}
S_{A_{i} A_{j}} & =\frac{1}{m-1} \sum_{k=1}^{m}\left(f_{i}\left(u_{k}\right)-\mu_{i}\right)\left(f_{j}\left(u_{k}\right)-\mu_{j}\right), \\
S_{A_{i}} & =\sqrt{\frac{1}{m-1} \sum_{k=1}^{m}\left(f_{i}\left(u_{k}\right)-\mu_{i}\right)^{2}},
\end{aligned}
$$




$$
S_{A_{j}}=\sqrt{\frac{1}{m-1} \sum_{k=1}^{m}\left(f_{j}\left(u_{k}\right)-\mu_{j}\right)^{2}},
$$

$\mu_{i}$ and $\mu_{j}$ are the respective mean value of $A_{i}$ and $A_{j}$.

During the process of calculating the Pearson's correlation coefficient, sometime the coefficient calculated may be no sense. So we consider hypothesis testing of a pair of attributes when we calculate the coefficient. In the hypothesis testing, if $\mathrm{p}-$ value $<0.05$, then the coefficient is significant and the coefficient calculated is acceptable, otherwise, the coefficient calculated is not acceptable. The revised correlation coefficient is as follow:

$$
R_{-} \operatorname{Cor}\left(A_{i}, A_{j}\right)= \begin{cases}\operatorname{Cor}\left(A_{i}, A_{j}\right) & \mathrm{p}-\text { value }<0.05 \\ 0 & \text { otherwise }\end{cases}
$$

Compared with the Pearson's correlation coefficient, the revised correlation coefficient is more suitable to represent the relationship between two attributes. Then, based on the extended reliability lifetime information table and the revised correlation coefficient, we use $R\left(A_{i} \mid A_{j}\right)$ to donate the coupled correlation matrix of the updated attributes ${ }_{i}$ and $A_{i}$ [5].Specifically,

$$
R\left(A_{i} \mid A_{j}\right)=\left(\begin{array}{ccc}
\eta_{11}(i \mid j) & \cdots & \eta_{1 D}(i \mid j) \\
\eta_{21}(i \mid j) & \cdots & \eta_{2 D}(i \mid j) \\
\vdots & \ddots & \vdots \\
\eta_{D 1}(i \mid j) & \cdots & \eta_{D D}(i \mid j)
\end{array}\right)
$$

Where $\eta_{p q}(i \mid j)=R_{-} \operatorname{Cor}\left(\left(A_{i}\right)^{p},\left(A_{j}\right)^{q}\right) \quad$ is the revised Pearson's correlation coefficient between $\left(A_{i}\right)^{p}$ and $\left(A_{j}\right)^{q}$. For instance, based on Table 2, the correlation matrix of attribute $^{A_{1}}$ and attribute $A_{2}$ is as follow:

$$
\begin{aligned}
& R\left(A_{1} \mid A_{2}\right)=\left(\begin{array}{ll}
\eta_{11}(1 \mid 2) & \eta_{12}(1 \mid 2) \\
\eta_{21}(1 \mid 2) & \eta_{22}(1 \mid 2)
\end{array}\right) \\
& =\left(\begin{array}{cc}
R_{-} \operatorname{Cor}\left(A_{1}, A_{2}\right) & R_{-} \operatorname{Cor}\left(A_{1},\left(A_{2}\right)^{2}\right) \\
R_{-} \operatorname{Cor}\left(\left(A_{1}\right)^{2}, A_{2}\right) & R_{-} \operatorname{Cor}\left(\left(A_{1}\right)^{2},\left(A_{2}\right)^{2}\right)
\end{array}\right),
\end{aligned}
$$

The coupled correlation matrix of attribute $A_{1}$ and reliability lifetime $A_{L}$ is as follow:

$$
R\left(A \mid A_{L}\right)=\left(\begin{array}{ccc}
\eta_{11}(i \mid L) & \cdots & \eta_{1 D}(i \mid L) \\
\eta_{21}(i \mid L) & \cdots & \eta_{2 D}(i \mid L) \\
\vdots & \ddots & \vdots \\
\eta_{D 1}(i \mid L) & \cdots & \eta_{D D}(i \mid L)
\end{array}\right)
$$

The value of revised correlation coefficient is in the range of -1 to 1 . Thus, we can observe the level of interplay between each pair of attributes and between each attribute and reliability lifetime. In this way, designers can get some helpful feedback information to improve the reliability of the products.

\section{CONCLUSION}

In this paper, based on the non-IIDness learning theory, we propose a scheme about reliability lifetime analysis. We mainly analyze the relationship between each pair of product attributes and the relationship between each attribute and reliability lifetime, where the attributes are continuous. According to the extended reliability lifetime information table and revised correlation coefficient, we donate the coupled correlation matrix of the updated attributes and reliability lifetime data. Then we can observe the level of interplay between each pair of attributes and between each attribute and product lifetime.

\section{REFERENCES}

[1] Cao, L., Non-IIDness Learning in Behavioral and Social Data. The Computer Journal, 57(9), pp. 1358-1370, 2014.

[2] Cui, W., A state-of-the-art review on fatigue life prediction methods for metal structures. Journal of Marine Science and Technology, 7(1), pp. 43-56, 2002.

[3] Zhang, X., Chen, X., Li, B., He, Z., Review of Life Prediction for Mechanical Major Equipments. Chinese Journal of Mechanical Engineering, 47(11), pp. 100-116, 2011.

[4] Wang, C., Cao, L., Wang, M., Li, J., Wei, W., and Ou, Y., Coupled nominal similarity in unsupervised learning. In CIKM 2011, pp 973-978, 2011.

[5] Wang, C., She, Z., Cao, L., Coupled Attribute Analysis on Numerical Data. IJCAI '13 Proceedings of the Twenty-Third international joint conference on Artificial Intelligence,pp 1736-1742, 2013.

[6] Cheng, X., Miao, D., Wang, C., Cao, L., Coupled Term-Term Relation Analysis for Document Clustering . In IJCNN2013, pp.1-8, 2013. 